Revue internationale P.M.E.

Économie et gestion de la petite et moyenne entreprise

\title{
PMI en situation d'insularité et marchés internationaux: le cas de la Corse
}

\section{Éric Faucon et Nadine Levratto}

Volume 7, numéro 2, 1994

URI : https://id.erudit.org/iderudit/1008388ar

DOI : https://doi.org/10.7202/1008388ar

Aller au sommaire du numéro

Éditeur(s)

Presses de l’Université du Québec

ISSN

0776-5436 (imprimé)

1918-9699 (numérique)

Découvrir la revue

Citer cet article

Faucon, É. \& Levratto, N. (1994). PMI en situation d'insularité et marchés internationaux: le cas de la Corse. Revue internationale P.M.E., 7(2), 31-58. https://doi.org/10.7202/1008388ar

\section{Résumé de l'article}

Cette étude vise à rendre compte de la manière dont les entreprises localisées dans des régions pouvant être définies comme des petites économies ouvertes peuvent trouver dans l'exportation une solution aux problèmes posés par I1étroitesse des marchés locaux. Après avoir mis en évidence la relative faiblesse des exportations réalisées par les PMI françaises et les difficultés éprouvées par ces firmes lors de l'élaboration des stratégies d'internationalisation, l'accent est mis sur les effets positifs à attendre des regroupements, formels ou informels, d'entreprises sur les échanges extérieurs. Les enseignements tirés de l'analyse de la situation nationale sont ensuite appliqués au cas d'une petite économie insulaire (la région corse), la restriction du domaine d'étude permettant 1) de confirmer l'intégration de l'exportation dans la stratégie globale des PMI et 2) la manière dont la coopération interfirme permet de surmonter le handicap du sous-dimensionnement. 


\title{
PMI en situation d'insularité et marchés internationaux: le cas de la Corse
}

\author{
Éric FAUCON * \\ Nadine LEVRATTO** \\ Université de Corse
}

\begin{abstract}
RÉSUMÉ
Cette étude vise à rendre compte de la manière dont les entreprises localisées dans des régions pouvant être définies comme des petites économies ouvertes peuvent trouver dans l'exportation une solution aux problèmes posés par l'étroitesse des marchés locaux. Après avoir mis en évidence la relative faiblesse des exportations réalisées par les PMI françaises et les difficultés éprouvées par ces firmes lors de l'élaboration des stratégies d'internationalisation, l'accent est mis sur les effets positifs à attendre des regroupements, formels ou informels, d'entreprises sur les échanges extérieurs. Les enseignements tirés de l'analyse de la situation nationale sont ensuite appliqués au cas d'une petite économie insulaire (la région corse), la restriction du domaine d'étude permettant 1) de confirmer l'intégration de l'exportation dans la stratégie globale des $P M I$ et 2) la manière dont la coopération interfirme permet de surmonter le handicap du sous-dimensionnement.
\end{abstract}

* Éric Faucon est chargé de relation avec les entreprises au CRITT (Centre régional d'innovation et de transfert de technologie) Corse-Technologie. Sa mission, assister les PME dans le domaine de la veille économique et technique, lui a permis d'acquérir une connaissance approfondie du terrain à partir de laquelle il a réalisé des travaux concernant la situation des entreprises en milieu insulaire. Adresse: CRITT Corse-Technologie, Université de Corse, Campus Grossetti, F 20250 Corte, France.

** Nadine Levratto est docteur ès sciences économiques, chargée de recherche CNRS au LATAPSES et chargée de cours à l'Université de Corse et dans le DESS d'ingéniérie financière de l'Université de Nice. Elle a publié des travaux relatifs 1) au fonctionnement du marché du crédit, abordé sous l'angle des théories du rationnement d'équilibre et de la firme bancaire, et 2) à la question du financement des entreprises et, en particulier, des PME françaises. Adresse : LATAPSES-CNRS/ UNSA, PEDRIC, Université de Corse, 250, rue Albert-Einstein, Sophia-Antipolis, F 06560 Valbonne, France. 


\begin{abstract}
The scope of this research is to exhibit how firms located in regions defined as small open economies may be induced to export in order to find an answer to the problems raised by the narrowness of local markets. Section I discusses the weak contribution of French SME's to export trade, and shows that it is mainly explained by obstacles in the settlement of international strategies. We suggest then how inter-firm co-operation may remove the international entry barriers related to firms size. In section II, the proposals which result from the examination of national data are applied to the Corsican Region, considered as a reasonable approximation of Small Island Economies. Restricting our field of analysis permits 1) to confirm the assumption that in SME's, exports cannot be considered as specific activities but are integrated into the general growth process of the firm and 2) to better understand how network organisation increases the efficiency of exporters.
\end{abstract}

\title{
RESUMEN
}

El objectivo de este trabajo es de dar cuenta como las empresas situadas en las regiones que se pueden definir como pequeñas economias abiertas, pueden encontrar en la exportación una solución a los problemas que ponen la pequeñez de los mercados locales. En la primera parte se demuestra la relativa insuficiencia de las exportaciónes realisadas por las PME francesas y las dificultades que tienen para elaborar estrategias de internacionalizatión. Insistimos sobre los efectos positivos sobre el comercio exterior que pueden dar los agrupamientos, formales o informales, de las empresas. Las lecciones de la situación nacional estan aplicadas al caso de una pequeña economía insular (Región de Corsica). La restrición del campo d'estidio permite 1) de confirmar la integración de la exportación en la estrategia global de las empresas $y, 2)$ de comprender como la cooperación inter-empresas ameliora la eficacia de los exportadores.

\section{Introduction}

«En dehors du cas spécifique de l'entreprise multinationale, dont le développement spatial a été longuement étudié, les difficultés que rencontre l'entreprise dans les rapports qu'elle entretient avec son espace de travail n'ont fait l'objet... que d'une attention marginale de la part des gestionnaires. » C'est en ces termes que G. Koenig (1989) commence l'article consacré aux décisions spatiales de l'entreprise de l'Encyclopédie de gestion. Il consacre ainsi l'idée selon laquelle la taille constitue un facteur décisif dès lors qu'il s'agit de rendre compte des processus de localisation des firmes et, par conséquent, des rapports qu'elles entretiennent avec leurs partenaires installés sur d'autres sites ou dans d'autres régions. La reconnaissance de ce phénomène de discrimination fondée sur la taille des unités de production nous conduit ici à retenir comme champ d'analyse le cas des entreprises de dimension petite et moyenne. En analysant les 
stratégies d'internationalisation des PMI installées dans des petites économies ouvertes, nous montrerons comment ces firmes sont contraintes de s'ouvrir vers l'extérieur si elles veulent atteindre un objectif de croissance, voire de maintien, de leur part de marché. Transparaît ici l'idée selon laquelle, l'ouverture internationale des PME ne se présente pas comme une action spécifique menée dans le cadre de services d'exportation qui, souvent, n'existent pas en tant que tels, mais s'inscrit dans une perspective de développement global de l'entreprise (Joyal et Ramangalahy, 1993).

Vouloir parler de l'insertion des PMI dans les échanges internationaux peut toutefois paraître surprenant, compte tenu des modestes performances qu'elles réalisent dans ce domaine. Néanmoins, la déclaration d'absence à priori des PME dans le commerce international repose sur une vue partiale et limitée d'après laquelle les échanges extérieurs relèvent du commerce entre les nations. Il n'est pas étonnant que l'essentiel des PME n'y figurent pas, puisqu'elles sont confinées dans les régions. De même que l'analyse conventionnelle du commerce extérieur renvoie aux grands espaces, elle n'appréhende que les firmes de grande dimension. Cette prise de position se trouve justifiée par le rôle déterminant joué par l'effet de taille en tant que barrière à l'entrée dans les flux d'échanges internationaux. Dans le but de se démarquer de tels a priori, nous adoptons ici une approche régionale du commerce extérieur ${ }^{1}$ grâce à laquelle peut être appréhendé le rôle international effectivement joué par les entreprises de moins de 500 salariés. Pour ce faire, il faut au préalable souligner le caractère inadapté des outils conceptuels traditionnels des théories du «commerce international » : soit que les outils se basent sur la notion d'avantages comparatifs pour conclure aux bienfaits de la spéculation totale, soit qu'ils s'appuient sur le théorème d'Hecksher-Ohlin-Samuelson, qui met l'accent sur la rareté relative des facteurs. Dans ce dernier cas, on aboutit à la mise en évidence du caractère optimal d'une spécialisation, cette fois incomplète, sans pour autant remettre en cause la nécessité d'une large échelle de production ${ }^{2}$. Ce caractère englobant de l'analyse du commerce international transparaît également au niveau des statistiques, lesquelles se caractérisent par l'utilisation de soldes nationaux dont la dimension macro-économique est évidente (tel est le cas des soldes d'échange, des taux de couverture ou de pénétration...).

Au terme de la précédente série de restrictions, il nous paraît donc important de préciser les bornes de notre domaine d'investigation, dont le but réside dans la mise en évidence de la place des PME et, plus particulièrement des plus

1. Pour un exposé des caractéristiques de cette démarche, voir Giraud (1991).

2. Une présentation exhaustive de ces deux approches et des critiques, notamment empiriques, auxquelles elles ont donné lieu est proposée par Muchielli (1987). 
petites d'entre elles, dans le commerce extérieur français. Afin de respecter la dimension régionale de notre approche, nous retiendrons ici le cas des économies insulaires dont l'analyse peut servir de base à l'établissement d'une connaissance générique de la situation dans laquelle se trouvent l'ensemble des territoires qui, du fait de leurs dimensions restreintes, possèdent un potentiel d'échange limité. À cet égard, le cas de la région de la Corse se révèle être un objet d'étude idéal et représentatif de toutes les petites économies ouvertes qui possèdent, entre autres, les caractéristiques suivantes :

- un tissu industriel essentiellement constitué de PME;

- une demande locale étroite et supérieurement bornée;

- une activité dominée par quelques secteurs.

Alors que l'économie expérimentale connaît un succès grandissant (Hey, 1992 ou Loomes, 1989), nous disposons ici d'un espace qui présente l'avantage de ne comporter que des entreprises de moins de 200 salariés, chose exceptionnelle en Europe. L'exploitation des séries de données publiées sur le tissu industriel corse, dans le cadre de la seconde partie de ce travail, nous permettra ainsi de donner une dimension appliquée aux considérations théoriques dont la présentation fera l'objet de la première partie de notre recherche.

\section{Les stratégies des PMI dans un environnement contingenté}

Il est devenu courant de souligner le retour en force des entreprises de petite et moyenne dimension et le rôle actif qu'elles ont à jouer en matière de croissance et d'emploi. Cependant, la connaissance de leurs comportements stratégiques reste, paradoxalement, encore assez limitée. Bien que l'extrême diversité des situations rencontrées constitue une entrave à la réalisation de recherches opérationnelles sur ce sujet, il faut néanmoins tenir compte des regroupements possibles et recourir à l'établissement de typologies afin de rendre compte des options envisageables dans ce domaine. Retenons comme hypothèse de départ l'idée que les options stratégiques des PME se présentent avant tout comme des choix destinés à maximiser les chances de survie de l'entreprise (Saporta, 1989). Nous considérons alors, à l'instar de M. Marchesnay (1991), que pour la plupart des firmes de moins de 500 salariés, il s'agit de procéder à un choix entre, d'une part, la diversification limitée à un territoire régional, voire national, et, d'autre part, la spécialisation étroite, accompagnée alors d'une forte propension à l'internationalisation.

C'est à l'analyse de la seconde branche de l'alternative précédente que seront consacrés les prochains paragraphes dans lesquels la mise en évidence des stratégies d'internationalisation des PMI, des comportements et des perfor- 
mances de ces unités sur les marchés internationaux, ainsi que des contraintes qui pèsent sur leurs perspectives de développement à l'extérieur, se présente comme le principal objectif.

\subsection{Le poids des PMI françaises dans le commerce extérieur}

Alors que les GE représentent approximativement $4 \%$ des entreprises industrielles françaises, elles ont réalisé en 1989 les trois quarts des exportations directes de l'industrie (SESSI, 1991). De fait, l'essentiel des exportations provient d'un nombre restreint de très grandes firmes dont la stratégie est d'emblée conçue à un niveau international, ce qui laisse supposer que la petite taille constitue un handicap pour la pénétration de marchés étrangers, ainsi que le prouve le tableau suivant.

TABLEAU 1

Répartition des exportations industrielles selon la taille des entreprises

\begin{tabular}{cc}
\hline Taille & Pourcentage d'exportation réalisé \\
\hline $20 \leq$ effectif $<50$ & 3,1 \\
$50 \leq$ effectif $<100$ & 4,0 \\
$100 \leq$ effectif $<200$ & 5,4 \\
$200 \leq$ effectif $<500$ & 11,6 \\
$500 \leq$ effectif & 75,9 \\
\hline
\end{tabular}

Source : SESSI

La mise en évidence d'une relation inverse entre taille et contribution à l'exportation, qui apparaît comme un élément clé dans l'étude des relations internationales entretenues par les PMI françaises (Amar et Crepon, 1990), doit cependant être affinée par la prise en considération des entreprises dites non exportatrices, c'est-à-dire qui réalisent moins de $5 \%$ de leur chiffre d'affaires à l'exportation. En effet, leur proportion qui s'élève à $60 \%$ dans les PMI contre $19 \%$ dans les GE industrielles pèse sur leur taux moyen d'export $(17,6 \%$ en 1989 comparé aux $35,1 \%$ de la population des GE). Par conséquent, loin d'adopter un raisonnement portant sur les PMI prises dans leur globalité, il s'agit maintenant de diviser cette population en différents groupes afin de rendre compte des divers comportements et performances qui les caractérisent et d'exclure les firmes qui, réalisant moins de $5 \%$ de leur chiffre d'affaires à l'étranger, ne peuvent pas être considérées comme exportatrices. 
Si les études réalisées sur les typologies d'entreprises exportatrices (Auquier, 1980; Banon et Delbreil, 1990 ; Bricout, 1990 ou encore Francq, 1990) montrent, de manière unanime, la faiblesse des PME sur le terrain de l'exportation, une analyse plus fine rend compte du fait que, dans l'industrie, secteur caractérisé par une structure d'exportation diluée (Bricout, 1991), trois catégories méritent d'être distinguées (Maliverney, 1991):

1. Les petits exportateurs qui réalisent à l'étranger entre 5 et $20 \%$ de leur chiffre d'affaires. C'est dans cette catégorie que se rangent plus de la moitié des PMI.

2. Les exportateurs confirmés définis par une propension à exporter située entre 20 et $60 \%$ du chiffre d'affaires. Noyau dur de l'activité exportatrice française, ce groupe contient surtout des GE, mais il faut souligner que les PMI qui s'y trouvent réalisent près de $9 \%$ du total des exportations.

3. Les entreprises spécialisées en exportation vendent plus de $60 \%$ de leur production à l'étranger et il est surprenant de constater qu'un quart des exportations des PMI est réalisé par des firmes appartenant à ce sousensemble.

C'est probablement dans l'évolution des parts relatives de ces sousgroupes que l'on peut trouver une explication de la tendance à la hausse suivie par la contribution des PMI aux exportations industrielles qui est passée de $22,7 \%$ en 1986 à $24,1 \%$ en 1989 , le tableau 2 permettant de renforcer ce constat.

TABLEAU 2

Indices d'évolution des exportations en millions de francs courants

\begin{tabular}{rcccccccc}
\hline Export & $\mathbf{1 9 8 2}$ & $\mathbf{1 9 8 3}$ & $\mathbf{1 9 8 4}$ & $\mathbf{1 9 8 5}$ & $\mathbf{1 9 8 6}$ & $\mathbf{1 9 8 7}$ & $\mathbf{1 9 8 8}$ & $\mathbf{1 9 8 9}$ \\
\hline $20-49$ & 112,4 & 110,5 & 103,5 & 111,2 & 99,7 & 101,7 & 111,3 & 112,9 \\
$50-99$ & 111,0 & 108,8 & 105,9 & 117,0 & 98,0 & 101,5 & 113,4 & 120,6 \\
$100-199$ & 105,5 & 110,1 & 103,4 & 110,4 & 95,8 & 103,4 & 109,9 & 116,6 \\
$200-499$ & 114,0 & 109,2 & 113,8 & 110,8 & 98,6 & 100,7 & 113,6 & 117,4 \\
\hline $20-499$ & 111,1 & 109,6 & 108,6 & 111,6 & 97,9 & 101,6 & 112,4 & 117,1 \\
+500 & 113,9 & 112,9 & 114,5 & 105,4 & 99,3 & 104,6 & 111,3 & 110,7 \\
\hline
\end{tabular}

Source : SESSI

Les évolutions respectives des différentes classes de PMI tendent, en effet, à corroborer l'idée d'un repli sur le marché intérieur des entreprises exportatrices confirmées, ce phénomène étant relevé en 1985 par J. Maliverney (1991). Les moindres ventes des entreprises de cet ensemble s'étant produites dans un 
contexte de croissance globale des exportations, les compensations sont à chercher dans les deux autres classes d'exportateurs et, tout particulièrement, parmi les très grandes entreprises et les plus importantes des PMI. La concentration du système exportateur à laquelle on assiste en France n'est pas exceptionnelle : elle se retrouve également dans d'autres pays de la Communauté européenne (c'est le cas par exemple de l'Italie, voir Gnes et Varetto, 1990). Ce recentrage au profit d'un nombre réduit d'entreprises exportatrices traduit en fait une redistribution des rôles parmi ces dernières, les petits exportateurs se voyant écartés des marchés porteurs et sûrs, leurs ventes se faisant davantage en direction de pays risqués et sur des marchés dont la demande est appelée à se tasser.

La typologie de marché établie par l'INSEE (Lehoucq, 1992) fait en effet apparaître des segments suivant le dynamisme de la demande et permet de constater qu'en 1989 les GE étaient les mieux positionnées sur les marchés en croissance où elles réalisaient $37 \%$ de leurs exportations contre $16 \%$ pour les PMI. Par ailleurs, ces dernières exportent trois fois plus de produits à faible demande que de produits à forte demande, les proportions variant relativement peu dans le temps car, entre 1984 et 1989 , l'effort d'exportation réalisé par les PMI se répartissait de manière relativement homogène entre les produits à demande forte $(+54 \%)$, moyenne $(+65 \%)$ et faible $(+57 \%)$.

Aussi, même si les PMI tendent à accroître leurs ventes à l'étranger et leur potentiel d'exportation, ces efforts ne sont couronnés de succès que pour les plus grandes d'entre elles. N'est-il pas en effet frappant de constater qu'un tiers des PMI exportatrices sont filiales de groupes nationaux ou étrangers? Ces derniers contrôlent donc directement ou indirectement plus de la moitié des exportations en termes de chiffre d'affaires des PMI. La tendance à la concentration dans les exportations se manifestant de manière continue depuis 1985 , le caractère pérenne de ce phénomène nous conduit maintenant à nous interroger sur les causes de l'existence d'une apparente barrière à l'entrée faisant intervenir la taille de l'exportateur sur les marchés internationaux.

\subsection{Peut-on parler de barrières à l'entrée fondées sur un effet «taille» en matière d'exportations?}

À la question de l'existence d'un éventuel handicap dont souffriraient les PMI désireuses de diversifier leur clientèle en vendant une partie de leur production à l'étranger, les développements précédents nous permettent de répondre par l'affirmative. Peut alors être considéré comme un principe de base de notre analyse l'idée selon laquelle, toutes choses étant égales par ailleurs, une firme qui exporte est en moyenne plus grande qu'une entreprise qui écoule la totalité de sa production sur le marché national. Toutefois, reconnaître ce type de discrimination n'est pas suffisant lorsqu'on veut mener une étude sur les causes des 
difficultés rencontrées par les PMI désirant adopter une stratégie d'expansion internationale. Il devient donc nécessaire de faire appel à une méthodologie plus fine dont l'origine se trouve dans les travaux consacrés à la relation entre taille et R-D.

Nous nous fondons ici sur l'idée schumpeterienne (Schumpeter, 1954), selon laquelle une taille importante constitue un facteur propice à l'engagement de dépenses de R-D, ainsi que des développements appliqués auxquels elle a donné lieu (seront ici utilisés l'approche et les résultats de Cohen, Levin et Mowery, 1987). La principale conclusion à laquelle parviennent ces recherches peut s'exprimer de la façon suivante : la taille influence positivement la probabilité qu'une entreprise fasse de la R-D mais, parmi les firmes engagées dans ce processus, la taille ne constitue pas un facteur explicatif de l'intensité en matière de R-D.

Les statistiques dont on dispose en matière d'engagement et de performances à l'exportation des PMI françaises, ainsi que les études économétriques réalisées permettent de considérer comme possible une transposition des conclusions dégagées dans le domaine de l'économie de la technologie au cas des échanges internationaux. Dans ce but, partons de la constatation d'une relation directe entre la taille de la PMI et sa présence sur les marchés étrangers, telle que la décrit le tableau 3.

TABLEAU 3

Répartition par taille du ratio Export/CAHT

\begin{tabular}{cccccccc}
\hline & $\mathbf{2 0 - 4 9}$ & $\mathbf{5 0 - 9 9}$ & $\mathbf{1 0 0 - 1 9 9}$ & $\mathbf{2 0 0 - 4 9 9}$ & $\mathbf{+ 5 0 0}$ & Hors Tr.* & Total \\
\hline Export/CAHT & 9,7 & 14,7 & 19,0 & 23,4 & 35,1 & 33,6 & 28,3 \\
\hline
\end{tabular}

Source : SESSI

${ }^{*}$ Les entreprises Hors Tranche sont en général des donneurs d'ordre.

Puis, enrichissons notre connaissance de la situation en ajoutant à ces données globales des statistiques plus fines fondées sur l'appartenance sectorielle et le statut d'exportateur ou de non-exportateur de l'entreprise. Ces données sont contenues dans le tableau 4.

On constate ici le caractère dilué des exportations industrielles qui sont le fait d'une proportion relativement importante d'entreprises plutôt faiblement exportatrices. La concentration ne peut donc pas être considérée comme un facteur exerçant une influence positive déterminante sur le volume des ventes réalisées à l'extérieur du territoire national. Mais, au-delà de cette constatation négative, restent à mettre en évidence les facteurs explicatifs de l'exportation. 
Dans cette perspective, nous allons exploiter la partie droite du tableau 4 d'après laquelle le chiffre d'affaires des entreprises exportatrices est plus élevé que celui des entreprises non exportatrices. D'un point de vue méthodologique, cela nous conduit à scinder la question de la relation générale entre la taille de la firme et l'exportation en deux sous-problèmes dont l'un relève du choix d'exporter ou de ne pas exporter alors que l'autre fait référence aux performances à l'exportation.

\section{TABLEAU 4}

Performances des entreprises exportatrices et non exportatrices

\begin{tabular}{l|c|c|c|c}
\hline & $\begin{array}{c}\text { Pourcentage } \\
\text { d'entreprises } \\
\text { exportatrices }\end{array}$ & $\begin{array}{c}\text { Taux } \\
\text { d'exportation* } \\
\text { moyen }\end{array}$ & \multicolumn{2}{|c}{$\begin{array}{c}\text { Chiffre d'affaires moyen } \\
\text { des entreprises (en MF) }\end{array}$} \\
\cline { 4 - 5 } & & $\begin{array}{c}\text { non } \\
\text { exportatrices }\end{array}$ & exportatrices \\
\hline Biens de consommation & 35,7 & 22,9 & 9 & 43 \\
Biens d'équipement & 39,25 & 22,8 & 22 & 137 \\
Biens intermédiaires & 32,3 & 18,9 & 12 & 96 \\
Industries agro-alimentaires & 15,8 & 19,2 & 15 & 196 \\
\hline
\end{tabular}

Source : Centrale des Bilans-SUSE

* Le taux d'exportation se définit comme le rapport entre le chiffre d'affaires exporté et le chiffre d'affaires global.

L'analyse de la décision d'exporter ou de limiter ses ventes au marché national a notamment été effectuée par Bricout (1991) sur la base d'un modèle dichotomique faisant apparaître l'influence de la taille (appréciée par le chiffre d'affaires). Les régressions effectuées sur des données descriptives d'une dizaine de secteurs, hétérogènes en termes de concentration, montrent que la taille exerce une influence significativement positive sur la décision d'élargissement de la surface commerciale de la firme.

Globalement, les entreprises exportatrices ont donc un volume d'affaires plus important que leurs homologues non exportatrices, tout comme les entreprises qui réalisent un niveau d'investissement immatériel élevé présentent une surface économique plus large que celles qui ne le font pas. Ce résultat s'explique par l'importance des moyens nécessaires à la mise en œuvre de stratégies d'exportation qui requièrent au minimum d'acquérir un niveau suffisant d'informations coûteuses, car souvent privatives, et de mettre en place un réseau de distribution ou des contrats de diffusion. Supporter de tels coûts, non récupérables en cas d'échec, ne s'avère possible que pour une grande entreprise qui peut les financer grâce à l'existence préalable d'une large gamme d'activités ou de produits. 
L'externalisation de certains services peut être envisagée, notamment auprès d'établissements financiers spécialisés ou bien d'intermédiaires commerciaux ayant vocation de favoriser les échanges marchands avec l'étranger. Cependant, on doit mentionner que non seulement bon nombre de PME manquent d'informations relatives à l'existence même d'organismes d'interface mais, qu'en outre, l'externalisation de segments stratégiques comporte un coût que l'entreprise n'est pas forcément prête à supporter. À cette condition fondamentale viennent s'en ajouter d'autres qui, bien que de moindre importance, doivent néanmoins être remplies afin de passer du désir à l'action d'exporter. Entrent dans cette catégorie certaines données financières telles que le ratio d'immobilisation qui intervient négativement de manière significative et le taux de capitaux permanents qui joue un rôle positif. En comparant ces résultats aux données bilantielles des PMI françaises, on comprend les raisons pour lesquelles ces entreprises sont si peu présentes sur les marchés extérieurs. D'une part, le niveau élevé du besoin en fonds de roulement des unités de dimension petite et moyenne dû à la lourdeur de leur cycle d'exploitation et à l'importance du crédit interentreprises nuit à la constitution d'un actif circulant élevé. D'autre part, la déstructuration du haut de bilan ainsi que le poids élevé de l'endettement à court terme constitue la caractéristique principale des PME nationales ${ }^{3}$. Ainsi, une taille suffisante et un niveau minimal de capitaux permanents conditionnent effectivement l'entrée des firmes sur le marché international; mais qu'en est-il de la relation entre les performances à l'exportation et la taille ?

Étudiant la seule population des entreprises exportatrices, il est possible de déterminer les facteurs influençant les performances réalisées par ces dernières. Les études réalisées (Bricout, 1990 et 1991) partent de la constatation d'une relation non linéaire entre l'effectif salarié ou le chiffre d'affaires - comme variables représentatives de la taille d'une entreprise - et le taux d'exportation réalisé. À supposer que l'on puisse parler de taille optimale ${ }^{4}$, celle-ci se situerait entre 120 et 200 salariés ou entre 130 et 200 millions de francs de chiffre d'affaires.

La recherche des variables micro-économiques ou sectorielles explicatives des performances à l'exportation des firmes ne fait pas apparaître d'influence significative du facteur taille. En revanche, peuvent être repérés des éléments exogènes permettant de tracer le profil des entreprises les mieux placées sur les marchés extérieurs. Est ainsi mis en évidence un effet positif du salaire moyen, utilisé comme indicateur du niveau de qualification (donc de savoir-faire) du

3. Pour une présentation détaillée de ces éléments, cf. Levratto (1993).

4. Des discussions sur cette notion peuvent être trouvées dans Audretsch (1992) et dans Cette et Szpiro (1992). 
personnel et de la productivité apparente du travail (mesurée par les ratios «valeur ajoutée par personne» ou «chiffre d'affaires par personne») qui rend compte simultanément de l'efficacité du mode de production utilisé et du caractère plus ou moins porteur du segment de marché occupé. Ce sont ainsi les entreprises situées sur des créneaux étroits de haute technologie et faisant appel à du personnel très qualifié qui exportent le plus, ces facteurs faisant office de barrières à l'entrée sur le marché international. En revanche, la part des salaires et des charges sociales dans la production exerce un effet négatif sur la performance des firmes à l'étranger.

A priori et compte tenu des enseignements précédents, l'influence de la taille sur la performance à l'exportation semble réduite. Toutefois, il s'agit d'être circonspect lors de l'interprétation de tels résultats, ne serait-ce qu'en raison de l'élimination de fait des plus petites PME de la population étudiée. De plus, les facteurs explicatifs semblent susceptibles d'intégrer implicitement un effet taille. Le salaire moyen est plus faible dans les PME qui emploient en outre un personnel moins qualifié (SESSI, 1991); ensuite, la productivité apparente du travail est plus élevée dans les GE; et enfin, les coûts salariaux et sociaux sont inversement liés à la taille de la firme.

Même s'il nous semble raisonnable de considérer que les PME exportatrices sont tout aussi performantes que leurs homologues de grande taille une fois franchies les limites du territoire national, il importe de garder à l'esprit l'existence d'une possible multicolinéarité entre les diverses variables explicatives retenues et la taille qui contribuerait à réduire le coefficient associé à cette dernière.

\subsection{Propositions en vue du renforcement du potentiel d'exportation des PMI régionales}

Ce travail ne se présente pas comme un programme de recherche-action trouvant sa source dans les recherches visant à donner un contenu théorique à la notion de PME et s'achevant avec la mise en place de mesures contribuant à donner à ces firmes la place qu'elles devraient idéalement occuper dans la structure économique française. Néanmoins, les constats précédents, qui concourent à mettre en évidence la difficulté d'insertion des PMI dans les échanges internationaux, soulèvent la question des moyens mis en œuvre pour réduire un tel handicap.

Sur le vu du tableau 5, il pourrait apparaitre rationnel de prôner une intégration des PMI dans des groupes, cette absorption leur garantissant une meilleure assise financière et un accès plus aisé à l'information. Un tel dirigisme n'est évidemment plus de mise essentiellement en raison des effets néfastes qu'il 
TABLEAU 5

Positionnement des PMI à l'exportation selon l'origine du capital

\begin{tabular}{|c|c|c|c|c|c|c|}
\hline & \multirow{2}{*}{\multicolumn{2}{|c|}{$\begin{array}{c}\text { PMI } \\
\text { indépendantes }\end{array}$}} & \multicolumn{4}{|c|}{ PMI filiales de groupes } \\
\hline & & & \multicolumn{2}{|c|}{ français } & \multicolumn{2}{|c|}{ étrangers } \\
\hline & 1 & 2 & 1 & 2 & 1 & 2 \\
\hline Biens intermédaires & 84,9 & 42,4 & 7,6 & 23,2 & 7,4 & 34,4 \\
\hline Biens d'équipement & 82,0 & 43,3 & 7,6 & 19,8 & 10,4 & 36,9 \\
\hline Biens de consommation & 89,0 & 58,2 & 5,3 & 19,4 & 5,7 & 22,5 \\
\hline Total & 85,7 & 47,4 & 6,7 & 21,0 & 7,5 & 31,6 \\
\hline
\end{tabular}

Source: SESSI

1. Proportion par rapport à l'ensemble des PMI (en \%).

2. Proportion des exportations des PMI du secteur (en \%).

peut produire en termes de bureaucratie et d'adhésion aux objectifs de la firme (Williamson, 1975). Il faut donc respecter le souci d'indépendance manifesté par de nombreux dirigeants de PMI qui ne considèrent pas leur unité comme une future GE. Tenir compte de l'organisation interne de la firme constitue donc un préalable nécessaire à l'analyse des rapports qu'elle entretient avec les marchés externes en général (Favereau, 1989) et internationaux en particulier.

Conformément aux principes précédents, il s'agit de rendre compte des stratégies d'internationalisation possibles pour les PME, ces stratégies devant à la fois préserver la petite taille et l'indépendance des firmes qui les adoptent, ainsi que leur capacité de réponse aux changements affectant les marchés. Deux voies complémentaires seront donc mentionnées, puis feront l'objet d'un examen empirique à partir du cas des entreprises localisées en région corse.

1. Contourner la contrainte exercée par la taille en procédant à des regroupements «volontaires» de firmes. De telles stratégies d'agglomération ne vont pas sans évoquer les notions de districts et de réseaux si souvent mentionnées en économie industrielle. De fait, en matière d'exportation, il semble envisageable d'avoir recours à de telles procédures qui reposent largement sur les relations que des PMI doivent entretenir avec des entreprises de service. La notion de chaîne export (Léo, Monnoyer-Longe et Philippe, 1991 et Léo, 1993) relève d'une telle logique, car elle intègre non seulement les flux de produits, mais également les informations (de fonctionnement et de développement) indispensables à la maîtrise des développements futurs des ventes à l'étranger. Ces chaînes se présentent ainsi comme des «structures 
molles» qui, fonctionnant selon un principe similaire à celui des réseaux locaux d'innovation (Torre, 1993) ou des districts, préservent l'indépendance des unités qui y participent tout en favorisant la constitution et le maintien d'un système de contacts extérieurs, ainsi que l'intensification de la communication avec le milieu économique.

L'association de firmes peut également se réaliser de manière apparemment plus fortuite, ainsi que le montrent les recherches mettant l'accent sur le rôle joué par les milieux territoriaux industrialisants (Beauviala-Ripert et Rousier, 1991). Les régions urbaines connaissent en effet un avantage évident en matière de relations avec l'étranger qui repose sur l'existence d'une structure capable de jouer le rôle d'incubateur en raison de la large gamme de services et de savoir-faire qu'elles assurent aux PMI qui s'y implantent (Storper, 1986).

2. Intensifier la spécialisation et bénéficier d'un quasi-monopole sur des parts de marché très réduites. La production de petites séries est considérée comme un atout pour les petites et moyennes entreprises du secteur industriel qui répondent aux attentes des GE dans lesquelles se généralise la production en flux tendus. Le maintien de cet avantage se révèle difficilement compatible avec la fabrication de grandes séries qui, reposant sur l'obtention d'économies d'échelle, laissent peu de place à la différenciation des produits. Le choix de la flexibilité accrue requiert toutefois le respect de deux conditions, l'une relevant de l'organisation des processus de production, et l'autre faisant référence aux marchés sur lesquels les entreprises diffusent leurs produits.

L'utilisation d'une main-d'œuvre qualifiée doit constituer l'un des objectifs visés par les PMI soucieuses de leur insertion dans les échanges internationaux. Afin d'opposer des arguments à l'encontre de l'intensité capitalistique élevée, caractéristique des GE qui développent des sites de production monoproducteurs, les PMI doivent se doter d'un facteur travail flexible garantissant une meilleure adaptabilité aux modifications de la demande exprimée par d'autres firmes ou par les ménages. Plus que de développer une large gamme de produits, il s'agit dans ce cas de mener à son terme l'évolution d'un produit sur un marché de plus en plus segmenté, car une telle stratégie repose davantage sur une approche du bien en termes de caractéristiques (Lancaster, 1975) que sur le seul terme du prix. Un tel choix ne demeure pas sans effets sur les spécificités des marchés sur lesquels les PMI peuvent se positionner en tant qu'offreurs performants. Pour être concurrentielles, les entreprises de taille petite et moyenne doivent exploiter les créneaux laissés vacants par les GE. Ces espaces se révèlent parfaitement adaptés à la mise en valeur de petites séries de qualité dont la production fait largement appel à la flexibilité et à la souplesse, éléments indispensables pour aborder la fluidité des échanges (Giraud, 1991). Il est à ce 
propos frappant de constater le regain d'intérêt suscité par la notion de «créneaux de marché », actuellement largement exploité dans le cadre de travaux sur l'émergence des firmes et des nouvelles technologies.

À l'issue de cette première partie consacrée à la mise en évidence des caractéristiques des PMI confrontées à la nécessité d'exporter, il nous apparaît nécessaire de rappeler que le commerce extérieur français repose largement sur des relations de proximité géographique, ainsi que l'atteste le bon classement des régions frontalières. On peut alors s'interroger sur les perspectives d'insertion des régions périphériques, d'une part, et des régions qui ne possèdent aucune frontière terrestre avec d'autres pays, d'autre part. Apporter un éclairage sur leur position sera l'objet de la prochaine partie consacrée à l'étude d'un espace insulaire : la Corse.

\section{La croissance des exportations corses: une conséquence de l'évolution des stratégies des PMI locales}

La région peut être définie comme «un territoire formant un ensemble économique, un marché régional déterminé non seulement par une interaction mécanique du milieu physique et du milieu humain, mais par l'activité économique des hommes, c'est-à-dire par ses relations avec des territoires périphériques ou plus éloignées... » (Lajugie, Delfaud et Lacour, 1985). L'analyse qui suit se situe donc résolument dans le champ de l'économie régionale, la Corse correspondant tout à fait à la définition de région proposée ici avec, de plus, l'existence d'un recoupement total entre l'espace géographique et l'espace économique. Cette île présente en effet des caractéristiques économiques telles qu'il est possible d'y étudier finement les stratégies d'internationalisation des PMI. L'analyse qui précède montre que l'effet taille joue un rôle non négligeable dans la prise de décision d'exporter; or, les entreprises locales présentent la caractéristique d'être toutes en dessous du seuil des 200 salariés et, pour une grande part d'entre elles, d'employer moins de 10 personnes. Si l'on considère comme acquis le handicap à l'exportation suscité par une faible taille, l'étude des moyens mis en œuvre pour le compenser se révèle particulièrement intéressante. La connaissance des caractéristiques du milieu industriel local, qui peut être abordée à travers une présentation rapide des différents secteurs industriels, est une base nécessaire à la compréhension du comportement des acteurs microéconomiques. De même, l'implication des PMI dans les stratégies d'internationalisation peut être évaluée à partir des échanges extérieurs de l'île et de leur évolution au cours du temps. À partir de ces éléments d'ordre général, il est possible de mener une réflexion visant à déterminer les facteurs permettant de déclencher une dynamique d'exportation au sein des PMI. Ce travail peut 
retenir les directions définies à partir d'hypothèses émises dans le cadre de travaux concernant des analyses plus globales du comportement des entreprises industrielles, telles que celles de R. Ratti et M. Baggi (1991) pour qui existe une corrélation positive entre le degré d'innovation et le degré d'ouverture du secteur régional industriel. La stratégie des PMI sera donc examinée sous cet angle, puis y seront intégrés des aspects connexes tels que les accords interfirmes, les stratégies de concentration ou les accords de sous-traitance.

\subsection{Un tissu économique dominé par la TPE et les activités orientées vers la demande finale}

La situation du tissu industriel corse est caractérisée par l'absence d'entreprises de taille importante ou moyenne. En effet, comme le montre le tableau cidessous, aucune entreprise industrielle n'emploie plus de 200 salariés.

\section{TABLEAu 6}

Répartition des unités industrielles corses par tranches d'effectifs salariés et secteurs d'activités

\begin{tabular}{lrrrrrrrrr}
\hline & \multicolumn{8}{c}{ Tranches d'effectifs salariés } \\
& Total & $\mathbf{0}$ & $\mathbf{1 - 2}$ & $\mathbf{3 - 5}$ & $\mathbf{6 - 9}$ & $\mathbf{1 0 - 1 9}$ & $\mathbf{2 0 - 4 9}$ & $\mathbf{5 0 - 9 9}$ & $\mathbf{1 0 0 - 1 9 9}$ \\
\hline Industries & & & & & & & & & \\
agro-alimentaires (IAA) & 537 & 210 & 172 & 94 & 32 & 22 & 6 & 1 & 0 \\
Énergie & 39 & 29 & 2 & 2 & 0 & 0 & 0 & 3 & 0 \\
Intermédiaires & 225 & 86 & 54 & 33 & 21 & 19 & 12 & 0 & 0 \\
Équipement & 233 & 109 & 56 & 41 & 13 & 9 & 4 & 0 & 1 \\
Biens de consommation & & & & & & & & & \\
courante (BCC) & 502 & 265 & 147 & 56 & 17 & 12 & 4 & 0 & 1 \\
Total & 1536 & 699 & 431 & 226 & 84 & 63 & 27 & 4 & 2 \\
\hline
\end{tabular}

Source: INSEE, Fichier SIRENE 1991

Au-delà de l'absence de grandes entreprises, l'analyse du tissu montre que les firmes occupant moins de deux salariés représentent les sept dixièmes des unités existantes. Cette situation est exceptionnelle en France, aucune autre région ne présentant un tissu industriel exclusivement composé d'entreprises de petite dimension.

La nomenclature d'activités et de produits (NAP) 15 permet d'observer de façon plus fine la situation des PMI dans les cinq secteurs industriels suivants: les industries agro-alimentaires (IAA), l'industrie des biens de consommation 
courante (BCC), l'industrie des biens intermédiaires et l'industrie des biens d'équipement ${ }^{5}$. Avant d'examiner chaque secteur industriel, il convient d'observer que les secteurs IAA et BCC regroupent les deux tiers des unités industrielles.

\subsubsection{Les industries agro-alimentaires}

Les IAA, dont la production est assurée par 537 unités, se présentent, à priori, comme un secteur extrêmement peu concentré, cette caractéristique contrastant avec ce que l'on peut observer sur le plan national ou européen. En effet, sur le total des unités répertoriées, 488 comptent moins de 10 salariés, ce qui permet de les classer dans la catégorie des TPE (très petites entreprises), dont la vocation consiste moins à conquérir des parts importantes de marché à l'extérieur qu'à assurer la satisfaction d'une demande exprimée à l'échelle microrégionale. La répartition des 49 entreprises restantes est loin d'être homothétique. Au contraire, plus on avance dans les classes d'effectifs, plus le nombre de firmes est faible, aucune entreprise de la région n'employant plus de 100 salariés.

\subsubsection{L'industrie des biens de consommation}

Secteur comptant 501 entreprises, l'industrie des biens de consommation se révèle largement dispersée, 445 unités employant moins de 5 salariés. Outre la forte densité de la répartition dans les deux premières classes d'entreprises, on constate, ici encore, qu'une seule unité de fabrication utilise une main-d'œuvre supérieure à 100 personnes. Doit également être signalé le fait que les industries des biens de consommation connaissent des taux d'encadrement très faibles - autour de $9 \%$ - ce qui, ajouté à leur taille, les rend peu performantes pour la compétition extérieure.

\subsubsection{L'industrie des biens d'équipement}

Quoique dispersée, la distribution des unités de production des biens d'équipement se caractérise par un éparpillement moindre que celui associé à l'industrie des biens de consommation, cette structure pouvant être expliquée par la nature des biens produits. En effet, les processus de production ici nécessaires sont plus lourds et plus complexes que ceux requis par la fabrication de biens de consommation courante. Ainsi, 165 unités emploient moins de deux salariés et

5. La production et la distribution d'énergie ne seront pas traitées dans ce travail, car elles relèvent d'un monopole d'État. Le fonctionnement et les stratégies observés dans ce secteur ne concernent donc pas les analyses en tant que PMI. 
présentent donc un caractère strictement artisanal. Seules les cinq entreprises de la branche aéronautique ont une activité qui leur permet d'avoir des échanges internationaux denses. Ces unités emploient une main-d'œuvre qualifiée, d'une part, et requièrent l'utilisation de technologies de pointe et d'outils de production compétitifs, d'autre part. L'analyse de ces entreprises appelle donc le même commentaire que précédemment: leur petite taille constitue un handicap pour l'accès aux marchés internationaux.

\subsubsection{L'industrie des biens intermédiaires}

Ce secteur comporte trois sous-groupes, qui sont: les industries extractives, la transformation métallique et non métallique et, enfin, la chimie et la parachimie. Parmi les industries extractives, seule la production de minéraux divers est représentée sur la région avec deux unités qui présentent l'intérêt d'être exportatrices. En outre, le secteur de la production de matériaux de construction est important avec 105 entreprises, mais reste totalement tourné vers la demande régionale.

Parmi les industries de la transformation métallique et non métallique, les unités appartenant au secteur du travail des métaux sont au nombre de 88 et présentent une taille plutôt élevée en regard de la norme régionale. Quelquesunes de ces entreprises ont réussi à atteindre un niveau de compétitivité qui autorise leur présence sur les marchés internationaux. Les quatre autres secteurs de la NAP 100 appartenant à l'industrie de la transformation métallique et non métallique ne sont constitués que d'un petit nombre d'entreprises ( 8 au total) qui apparaissent toutes dans les tranches inférieures à 9 salariés. On peut déduire de cette configuration le caractère marginal de ces activités.

La chimie et la parachimie constituent le dernier sous-groupe de l'industrie des biens intermédiaires. Ce dernier secteur est faiblement développé et constitué de firmes de très petite dimension.

Au terme de cette présentation de l'industrie corse, il convient de retenir deux traits marquants :

1. Les entreprises sont de très petite taille et souffrent donc des problèmes suivants : taux d'encadrement généralement bas, mauvaise maîtrise du marché, difficultés d'accès à l'information et absence d'économies d'échelle.

2. La production industrielle répond fréquemment à un besoin régional, voire microrégional ; elle existe pour des raisons de proximité et elle est massivement tournée vers des biens connaissant une faible demande, donc peu recherchés sur le plan international. 
Cette présentation de l'industrie de l'île par grands secteurs pourrait laisser croire que les PMI corses ne sont pas concernées par l'internationalisation. Néanmoins, une analyse de la balance commerciale corse et de son évolution au cours du temps permet de nuancer ce propos.

\subsection{Une évolution favorable des exportations de l'île}

La balance commerciale de la Corse laisse apparaitre un déficit important tant en volume qu'en valeur, ainsi qu'on peut le constater sur le tableau suivant.

\section{TABLEAU 7}

Balance commerciale de la Corse 1982-1992

\begin{tabular}{cccc}
\hline Années & Importations & Exportations & Taux de couverture \\
\hline 1982 & 175798 & 59240 & $33,69 \%$ \\
1983 & 207730 & 64529 & $31,09 \%$ \\
1984 & 247025 & 77465 & $31,35 \%$ \\
1985 & 252984 & 75165 & $29,71 \%$ \\
1986 & 327324 & 69655 & $21,28 \%$ \\
1987 & 347976 & 89824 & $25,65 \%$ \\
1988 & 377376 & 107276 & $28,42 \%$ \\
1989 & 366892 & 111597 & $30,42 \%$ \\
1990 & 409639 & 130502 & $31,85 \%$ \\
1991 & 447292 & 206853 & $46,25 \%$ \\
1992 & 435332 & 159836 & $36,72 \%$ \\
\hline
\end{tabular}

Source : Direction régionale des douanes, unité : milliers de francs.

L'étude en flux financiers du commerce extérieur de l'île appelle plusieurs commentaires :

1. Bien que déficitaires, les échanges extérieurs tendent à s'améliorer. En effet, le taux de couverture de l'année 1991 est de $46 \%$ contre 33,69\% en 1982. Ce taux s'établit à $36,72 \%$ en 1992 . Ce léger repli n'est pas une contre-performance, car il est dû à une situation conjoncturelle exceptionnelle en 1991. En effet, 1991 a été marquée par d'importantes ventes d'électricité et des cessions de matériel aéronautique d'occasion qui sont des opérations à caractère exceptionnel.

2. Les échanges internationaux de l'île s'accroissent au cours du temps avec une progression notable de $10 \%$ entre 1990 et 1992 . Le rééquilibrage de la balance commerciale est donc le fait d'une progression des exportations et non pas d'une réduction des importations. 
3. Les exportations progressent à un rythme plus important que celui des importations avec des taux d'accroissement de $15 \%$ entre 1989 et 1990 et de $22 \%$ sur la période $1990-1992$.

4. Conséquence de l'ensemble des phénomènes décrits ci-dessus, le déficit commercial semble se stabiliser depuis trois ans autour de $280 \mathrm{MF}$ alors qu'il progressait de façon importante les années précédentes.

Cette analyse globale des échanges extérieurs montre que la compétitivité des PMI de l'île s'améliore. Il est clair que les PMI se positionnent plus facilement sur le marché international. L'étude de la nature des produits exportés peut contribuer à fournir un éclairage sur l'amélioration des échanges entre la Corse et l'étranger.

\section{TABLEAU 8}

Importance relative des différents produits dans le total des exportations

\begin{tabular}{lcc}
\hline & $\mathbf{1 9 8 4}$ & $\mathbf{1 9 9 2}$ \\
\hline Produits agricoles & $56,8 \%$ & $45,6 \%$ \\
Produits des industries agro-alimentaires & $23,0 \%$ & $33,1 \%$ \\
Biens d'équipement & $1,8 \%$ & $2,4 \%$ \\
Biens intermédiaires & $2,6 \%$ & $3,3 \%$ \\
Biens de consommation courante & $0,6 \%$ & $6,5 \%$ \\
Énergie & - & - \\
Autres & $15,2 \%$ & $9,1 \%$ \\
\hline
\end{tabular}

L'observation de l'importance relative des différents postes d'exportation et de leur évolution entraîne différents commentaires :

1. Le poste produits agricoles perd beaucoup de poids relatif entre 1984 et 1992 au bénéfice des industries agro-alimentaires et des biens de consommation courante. La structure des importations semble donc glisser d'un système de type exportation de produits primaires non transformés vers un système où les exportations réalisées par les unités industrielles ont un poids plus significatif.

2. Certains postes ont une faible valeur relative qui évolue peu entre 1984 et 1992 mais, compte tenu de l'importante progression des exportations sur la période considérée, le chiffre d'affaires à l'exportation de ces industries s'est considérablement accru en valeur absolue. Tel est le cas pour l'industrie des biens de consommation courante qui réalisait un chiffre d'affaires export de $464 \mathrm{KF}$ en 1984 contre un chiffre d'affaires de $10301 \mathrm{KF}$ en 1992. 
3. L'évolution de l'importance relative des différents postes dans le total des exportations masque souvent des modifications importantes à l'intérieur de l'un ou l'autre des secteurs industriels. De ce point de vue, il convient d'apporter un éclairage sur les industries agro-alimentaires et celles de production des biens d'équipement.

En 1984, les exportations de produits agro-alimentaires étaient très largement dominées par les boissons et notamment le vin ; compte tenu de sa basse qualité et des restructurations importantes du vignoble, celui-ci devait perdre des parts de marché jusqu'en 1990, date à laquelle la politique de qualité a porté ses premiers fruits et a permis aux exportations de croître à nouveau. Dans le même temps, sur la période 1988-1992, la branche agro-alimentaire a connu la très forte croissance des activités aquacoles dont le chiffre d'affaires à l'exportation a été multiplié par six sur la période 1988-1992 passant de 7 à 49 MF. Aujourd'hui, ces activités dominent très largement la branche IAA.

Les industries de production des biens d'équipement ont connu également de profonds bouleversements. En effet, en 1984, les exportations étaient assurées essentiellement par une unité de production de machines agricoles qui a disparu et a été remplacée par des entreprises travaillant dans le secteur de l'aéronautique et de la mécanique de précision. Ces entreprises de haute technologie fabriquent des produits à forte valeur ajoutée pour lesquels le transport représente un coût marginal.

En conclusion, soulignons que les exportations de l'île ont connu de considérables modifications au cours de la dernière décennie. Celles-ci peuvent être décrites de la façon suivante: apparition de nouvelles entreprises exportatrices et de nouveaux produits, modification du poids relatif des différents produits dans les exportations et enfin, accroissement important des chiffres d'affaires réalisés à l'extérieur pour certaines entreprises ou produits. L'ensemble de ces transformations découle d'une modification de comportement des entreprises insulaires analysée ci-après.

\subsection{Des stratégies nouvelles pour conquérir les marchés extérieurs}

La connaissance exacte du tissu des entreprises exportatrices permet de mieux approcher les différentes stratégies utilisées pour se positionner sur les marchés extérieurs. On recense aujourd'hui $66 \mathrm{PMI}$ exportatrices en Corse, ce qui représente un peu plus de $4 \%$ de l'effectif total des firmes corses. Ce chiffre reste peu élevé, puisque la moyenne nationale au niveau des PMI est de $12 \%$ d'exportatrices. Néanmoins, un bon nombre d'entreprises ont relativement peu 
d'expérience sur les marchés extérieurs puisqu'elles n'ont commencé à exporter que ces deux dernières années. Cela tend à prouver que le nombre d'exportatrices devrait s'accroître à terme.

TABLEAU 9

PMI exportatrices par secteur d'activité

$\begin{array}{lr}\text { Industries agro-alimentaires } & 43 \\ \text { Biens intermédiaires } & 7 \\ \text { Biens d'équipement } & 6 \\ \text { Biens de consommation courante } & 10\end{array}$

Source : Direction régionale du commerce extérieur.

Les entreprises exportatrices se rencontrent massivement dans le domaine agro-alimentaire et plus précisément dans le secteur viticole. Les entreprises aquicoles ont également un poids non négligeable avec sept unités qui représentent la quasi-totalité de la branche aquicole de l'île, les unités en présence sont de plus considérablement impliquées dans le commerce extérieur, puisque huit sur dix d'entre elles ont des liens commerciaux avec l'étranger. Le reste des unités agro-alimentaires sont des conserveries (3), des fromageries (5), des biscuiteries (5) ou des charcuteries (1). L'ensemble de ces unités se positionnent sur des secteurs à faible demande et doivent sans doute adapter leurs stratégies à cette réalité.

Les entreprises exportatrices de biens intermédiaires se situent essentiellement dans le secteur de la pierre, dans la première transformation du bois et dans la production de matériaux de construction. La domination des carriers sur le secteur s'explique par la présence d'un quasi-monopoleur.

Le secteur des biens d'équipement est marqué par la forte présence des activités aéronautiques. Cependant, de nouvelles entreprises apparaissent, notamment dans le secteur de la fabrication d'équipement lourd destiné à l'industrie ou aux entreprises. L'entrée de ces PMI sur les marchés internationaux est significative de l'amélioration intervenue depuis deux ans dans la situation du commerce extérieur de l'île. Il convient enfin de se livrer à une rapide description des entreprises exportatrices de biens de consommation courante. Ces unités ont des productions très diverses, mais relèvent toutes de l'artisanat avec des tailles strictement comprises entre 0 et 3 salariés. Les entreprises exportatrices sont donc peu différentes de celles rencontrées dans l'ensemble de l'industrie régionale, puisque petites et majoritairement productrices de biens à faible demande. 
La petite taille de ces entreprises se trouve à l'origine d'un certain nombre de handicaps en termes de stratégies d'internationalisation. Il est possible de définir trois grandes catégories de handicaps liés à la structure même des entreprises:

1. Les PMI souffrent fréquemment d'un défaut de personnel d'encadrement. Or, la mise en place de stratégies commerciales orientées en direction des marchés étrangers nécessite un investissement immatériel important en termes de prospection et de mise en place de circuits de livraison et de mécanismes financiers.

2. L'exploitation de marchés extérieurs implique la détention d'informations préalablement au lancement des activités d'exportation et une veille continue par la suite. Les informations recherchées sont liées à l'évolution des réglementations, de la demande, mais également à l'action de la concurrence ou à la solvabilité des clients. L'accès à ces informations est souvent difficile pour des entreprises peu habituées à la veille et ayant mal identifié les lieux de collecte de l'information.

3. Les difficultés de trésorerie, fréquentes au sein des PMI, sont un obstacle supplémentaire. En effet, la prospection initiale de marchés entraîne souvent des dépenses considérables que les chefs d'entreprise hésitent à engager compte tenu d'une incertitude accrue relative au succès de ces opérations. Ces coûts sont souvent de nature irrécouvrable mais, même dans le cas contraire, leur amortissement implique la réalisation d'un chiffre d'affaires très élevé et l'entreprise n'est pas toujours structurée pour accroître sa production en conséquence.

Les structures financières et productives des PMI les contraignent à trouver des solutions novatrices et différentes de celles utilisées dans le monde de la grande entreprise pour se positionner sur les marchés internationaux. Ce phénomène se trouve encore accentué dans l'île où la faible taille des marchés et le surcoût lié au transport sont des facteurs contribuant à renforcer encore la situation défavorable en termes de coût des PMI. Ces firmes ne peuvent donc se positionner que sur des marchés où les GE sont absentes ou sur des créneaux de marché déterminés soit par une technologie spécifique, soit, plus généralement, par des éléments de différenciation du produit par rapport à d'autres produits identiques ou substituables. Dans le cas de l'île, on peut dénombrer cinq stratégies différentes utilisées par les PMI pour être compétitives face à leurs concurrentes étrangères.

\subsubsection{Les innovations de produits et de procédés}

Quelques entreprises ont choisi de s'orienter vers des activités à risque économique élevé compte tenu de l'inexistence sur le marché du bien produit et de la complexité liée à la maîtrise d'une technologie parfois naissante et aux contours 
évolutifs. Tel est le cas du secteur des produits de la mer. Après des débuts difficiles, les producteurs ont réussi à maîtriser les techniques fondamentales, puis ont progressivement développé un savoir-faire fondé notamment sur des transferts de procédés depuis la Norvège. La production aquicole est aujourd'hui exportée à $90 \%$.

Ce phénomène de transferts de procédés se retrouve également dans des secteurs tels que la mécanique de précision où l'acquisition de technologie s'est faite à partir de la formation des hommes. La technicité ainsi acquise et le soutien des pouvoirs publics ont permis de créer des entreprises dont certaines exportent jusqu'à $50 \%$ de leur production.

\subsubsection{Les stratégies qualité}

Certaines PMI ont choisi de différencier leur produit en lui conférant une qualité supérieure et en la faisant reconnaître par des instances officielles ou interprofessionnelles. Tel est le cas des produits agro-alimentaires où les transformateurs ont fait évoluer leurs produits vers des mentions qualité diverses: telles mentions biologiques ou appellations d'origine. Les stratégies qualité visent également les procédés avec la mise sous assurance qualité d'unités de production. Ces stratégies revêtent fréquemment une importance capitale au niveau des potentialités d'exportation.

\subsubsection{Les regroupements et les accords de coopération}

Afin de profiter d'effets de taille, les PMI se regroupent, ce qui leur permet d'amortir les frais liés à une stratégie d'exportation. Il existe plusieurs formes de groupements professionnels qui correspondent en fait à l'objectif retenu par les chefs d'entreprises. Les structures interprofessionnelles regroupant de nombreuses unités réalisent simultanément un travail sur la qualité du produit et une action commerciale. D'autres organisations ont pour seul but de centraliser une offre et de trouver les marchés correspondants. Ce sont fréquemment des sociétés de capitaux regroupant quatre ou cinq entreprises. Ces sociétés assurent la promotion d'une marque et du savoir-faire de leurs actionnaires.

Afin de se diversifier et d'étendre la gamme de leurs produits et savoirfaire, quelques entreprises ont choisi d'industrialiser des produits issus de recherches accomplies par des sociétés ou inventeurs localisés hors de l'île. Ces accords peuvent prendre différentes formes qui vont de la cession de licence ou de la concession de droits d'exploitation à l'industrialisation et la commercialisation en commun avec partage des risques et bénéfices. Ces accords sont particulièrement intéressants dans le cadre de stratégies d'internationalisation lorsqu'ils sont réalisés avec des sociétés étrangères car, dans ce cas, celles-ci 
deviennent à terme des points d'appui commerciaux. On dénombre trois accords de ce type dans le secteur de production des biens d'équipement et un dans le secteur de production des BCC. D'une manière générale, les regroupements d'entreprises permettent aux PMI de pénétrer sur les marchés extérieurs et de combler des lacunes qui, autrement, ne pourraient l'être. En effet, sans ce type de coopération, les PMI seraient certainement confinées au marché insulaire ou contraintes à la sous-traitance.

\subsubsection{Les concentrations et la sous-traitance}

Dans certains secteurs, des concentrations se sont opérées par le biais du rachat d'unités de production connaissant des difficultés financières. Ces rachats ont permis la constitution d'unités de taille suffisante pour positionner la production sur le marché international. Tel est le cas pour les industries de la pierre qui réalisent l'essentiel des exportations du secteur de production de matériaux de construction.

Quelques unités de production travaillent pour le compte d'entreprises de taille plus grande qui sont exportatrices. De tels accords permettent aux PMI locales de dépasser les barrières du marché régional, mais présentent l'inconvénient de nécessiter une forte spécialisation dont découle fréquemment une dépendance considérable vis-à-vis des donneurs d'ordre. Ces stratégies peuvent toutefois être porteuses dans le cas où les firmes sous-traitantes conservent une production en propre.

\section{Conclusion}

L'objet de ce travail visait à rendre compte de la place trop limitée que les PMI des petites économies ouvertes occupent dans les échanges internationaux et d'envisager les moyens mis en œuvre par ces unités et par les autorités publiques afin de pallier le faible potentiel de demande caractéristique des marchés régionaux où elles ont coutume d'intervenir. Les firmes de moins de 500 salariés localisées dans des régions périphériques de l'Europe souffrent de multiples handicaps qui sont encore plus lourds dans les «petites économies insulaires », à savoir des îles de moins d'un million d'habitants, politiquement dépendantes et présentant une forme de développement impulsé du dehors par une rente administrative ${ }^{6}$. Compte tenu des contraintes qui pèsent non seulement

6. Voir Poirine (1993) pour une présentation du modèle MIRAGE/ARABE décrivant les effets de l'existence d'une rente, y compris administrative, sur l'activité de ces économies. 
sur leur croissance, mais souvent sur leur survie, ces entreprises sont amenées à envisager l'exportation comme l'unique stratégie pouvant consolider leur position économique grâce à un élargissement de la part de marché occupée.

En étudiant le cas de la région corse, nous avons, d'une part, cherché à procéder à une analyse approfondie de la place occupée par les entreprises insulaires dans le commerce international. D'autre part, les caractéristiques de cette région peuvent contribuer à l'acquisition d'une connaissance générique de la situation des petites entreprises et, tout particulièrement, des TPE qui sont souvent les seules unités économiques privées rencontrées dans les régions européennes les moins favorisées (Régions d'objectif 1 : en retard de développement, d'objectif 2 : zones industrielles en déclin, d'objectif $5:$ zones rurales à développer). C'est sur l'apport de tels éléments de réflexion que repose le caractère heuristique de ce travail.

Toutefois, ces mêmes éléments se trouvent à l'origine de propositions normatives qu'il importera d'approfondir dans le futur. En effet, partant de l'idée que la petite taille des unités est le seul élément invariant lorsqu'on s'intéresse à la place internationale des PMI, la mise en œuvre de stratégies visant à atténuer les inconvénients qui en découlent se présente comme la seule option commerciale possible. C'est à l'aune de cet objectif que doivent donc être jugées les propositions suivantes:

- positionnement sur des créneaux qui, en raison de leur instabilité ne peuvent être occupés que par des PME auxquelles la petite taille confère une flexibilité nécessaire à l'obtention d'une rente sur des marchés volatils;

- développement des stratégies de coopération, non seulement dans le domaine productif, mais aussi en ce qui concerne les dépenses de R-D et les opérations commerciales à mener (Raines et Bachtler, 1993) ;

- partage des coûts d'informations qui se révèlent à la fois élevés et souvent irrécupérables sur des marchés caractérisés par l'existence d'asymétries entre les agents.

Si de tels facteurs peuvent figurer dans les objectifs des firmes de manière privative, il nous faut insister sur le rôle incitatif que peuvent jouer les organismes publics et parapublics tels que les Directions régionales du commerce extérieur, les postes d'expansion économique ainsi que la COFACE. Les dernières années ont d'ailleurs été marquées par une évolution des préoccupations majeures de ces institutions qui s'attachent à améliorer la situation des PMI (la simplification des modalités d'assurance prospection constitue une preuve de cette tendance). C'est dans la même logique que s'inscrivent les actions communautaires (Euromanagement, Europartenariat, Interprise...) visant à 
assurer un soutien aux PME actives en appuyant les projets de partenariat. Néanmoins, il est possible de considérer que les difficultés que les PMI rencontrent dans l'accès à l'information ne peuvent être atténuées que par l'émergence d'intermédiaires aux compétences multiples qui, outre une information technique, seraient également capables de distribuer des renseignements de nature économique, commerciale, juridique, voire culturelle.

\section{Bibliographie}

AMAR, M. et B. CREPON (1990), "Les deux visages de la concentration industrielle : efficacité et rente de situation », Économie et statistique, février, $n^{\circ} 229$, p. 14-19.

AUdRETSCH, D. (1992), «Small business in industrial economics: the new learning », Communication à l'École d'Été Méditerranéenne d'Économie Industrielle, Cargèse.

AUQUIER, A. (1980), «Sizes of firms, exporting behavior and the structure of French industry », Journal of Industrial Economics, décembre, vol. XLII, n 6, p. 256-273.

BEAUVIala-RIPERT, C. et N. ROUSIER (1991), "Milieux territoriaux internationalisant?», Revue d'Économie Régionale et Urbaine, nos 3-4, p. 178-194.

BANon, C. et M. Delbreil (1990), «Les entreprises françaises face au développement de leurs exportations », Document Banque de France.

BRICOUT, J.L. (1990), «Les entreprises exportatrices: une analyse sur données individuelles », Communication à la $15^{e}$ Journée des Centrales de Bilans, novembre.

BRICOUT, J.L. (1991), «Exporter: une question de taille», Économie et Statistique, juin, $\mathrm{n}^{\circ} 244$, p. $25-53$.

Cette, G. et D. SzPIRo (1992), «Rentabilité, productivité et taille de l'entreprise », Économie et Statistique, février, $n^{\circ} 251$, p. 57-74.

CoHEN, W.M., R.C. LeVIN et D.C. Mowery (1987), «Firm size and R-D intensity : a re-examination », Journal of Industrial Economics, juin, vol. XXXV, n ${ }^{\circ} 4$, p. 211-232.

FAVEREAU, O. (1989), «Marchés internes et marchés externes », Revue Économique, vol. $40, \mathrm{n}^{\circ} 2$, p. $273-327$.

FRANCQ, T. (1990), «Ouverture vers l'extérieur et performances à l'exportation des entreprises industrielles dans les années $80 »$, Économie et Prévision, $2^{\mathrm{e}}$ semestre.

GIBIAT, M. (1993), «Les modèles de la décision d'exporter en PME/PMI : synthèse des recherches depuis 20 ans ", Communication au Colloque international francophone de la PME, Carthage, Tunisie, 28-30 octobre. 
GIRAUD, G. (1991), «Les PME dans le commerce extérieur français : d'une proximité régionale à une proximité de délai », Revue Internationale $P M E$, vol. $4, \mathrm{n}^{\circ} 2$, p. 113-135.

GNES, P. et F. VARETTO (1990), «Industrie italienne et capacité compétitive sur les marchés internationaux », Communication à la $15^{e}$ journée des Centrales de Bilans, novembre.

HEY, J.D. (1992), «Experiments in industrial organization», dans A. Del Monte (éd.), Recent Developments in the Theory of Industrial Organization, Londres, Macmillan.

Joyal, A. et C. Ramangalahy (1993), «Typologie des comportements stratégiques des PME exportatrices: une étude de cas », Communication au Colloque international francophone de la PME, Carthage, Tunisie, 28-30 octobre.

JULIEN, P.A. (1990), «Vers une typologie multicritère des PME», Revue Internationale $P M E$, vol. 3, n 3 , p. 411-425.

KoENIG, G. (1989), «Décisions spatiales et gestion de l'entreprise », dans P. Joffre et Y. Simon, Encyclopédie de Gestion, Tome 1, Paris, Économica.

LAJUgie, J., P. Delfaud et C. LACOUR (1985), Espace régional et aménagement du territoire, $2^{\mathrm{e}}$ éd., Paris, Dalloz.

LANCASTER, K.J. (1975), «Socially optimal product differenciation» American Economic Review, mai, vol. 65, $\mathrm{n}^{\circ} 4$, p. 132-157.

LEHOUCQ, T. (1992), «Les PMI exportatrices », dans INSEE, La France des Entreprises.

LÉo, P.Y. (1993), «Le développement international des PME/PMI : outils pour une analyse stratégique », Communication au Colloque PME/PMI, Développement International, CER/GREPME, Aix-en-Provence, juin.

LÉo, P.Y., C. MonNoyer-Longe et J. PhilipPe (1991), «Métropoles régionales et PME : l'enjeu international », Revue d'Économie Régionale et Urbaine, $\mathrm{n}^{\circ} 1$, p. 41-70.

LEVRATTO, N. (1993), «L'influence des contraintes financières sur les performances des PME », Communication au Colloque francophone de la PME, Carthage, octobre.

LOOMES, G.C. (1989), «Experimental economics », dans J.D. Hey (éd.), Current Issues in Microeconomics, Londres, MacMillan.

MALIVERNEY, J. (1991), «Les entreprises exportatrices : une concentration accrue», Économie et Statistique, juin, ${ }^{\circ} 244$, p. 15-24.

MARCheSNAY, M. (1991), "Stratégies des petites et moyennes entreprises », dans R. Arena, J. De Bandt, L. Benzoni et P. Romani, Traité d'Économie Industrielle, Paris, Économica.

MuChielli, J.L. (1987), Principes d'Économie Internationale, Paris, Économica. 
POIRINE, B. (1993), «Le développement par la rente dans les petites économies insulaires », Revue Économique, novembre, p. 1169-1198.

RATTI, R. et M. BAGGI (1991), «Analyse stratégique et spatiale des accords de coopération entre entreprises du secteur industriel », Revue d'Économie Régionale et Urbaine, $\mathrm{n}^{\text {os } 3-4, \text { p. 465-478. }}$

RAINES, P. et J. BACHTLER (1993), «The use of cooperation agreements by small and medium-sized entreprises », dans Western Europe, Communication au 33rd Regional Science Association Congress, Moscou, 23-27 août.

SAPORTA, B. (1989), "Stratégies des petites et moyennes entreprises », dans P. Joffre et Y.Simon, Encyclopédie de Gestion, tome 1, Paris, Économica.

SChumpeter, J. (1954), Capitalisme, Socialisme et Démocratie, Paris, Payot.

Sessi (1991), Les Chiffres Clés des PMI, Paris, Dunod.

STORPER, M. (1986), «Technology and new regional growth centres », dans P. Nijkamp (éd.), Technological Change, Employment and Spatial Dynamics, Berlin, Springer Verlag.

TORRE, A. (1993), «Proximité géographique et dynamiques industrielles », Revue d'Économie Régionale et Urbaine, $\mathrm{n}^{\circ} 3$ «spécial proximité», p. 431-448.

Williamson, O.E. (1975), Markets and Hierarchies, New York, The Free Press. 